\title{
Eosinophilic esophagitis. What diet? How to treat it? The point of view of a gastroenterologist working in a department of allergology
}

\author{
Andrzej Kuźmiński ${ }^{1}$, Michał Przybyszewski ${ }^{1}$, Justyna Przybyszewska², Zbigniew Bartuzi ${ }^{1}$ \\ ${ }^{1}$ Department of Allergology, Clinical Immunology and Internal Diseases, Collegium Medicum in Bydgoszcz, Nicolaus Copernicus \\ University in Torun, Poland \\ ${ }^{2}$ Faculty of Health Sciences, Department of Nutrition and Dietetics, Collegium Medicum in Bydgoszcz, Nicolaus Copernicus \\ University in Torun, Poland
}

Gastroenterology Rev 2021; 16 (4): 318-323

DOI: https://doi.org/10.5114/pg.2021.105163

Key words: eosinophilic esophagitis, allergic diseases.

Address for correspondence: Andrzej Kuźmiński PhD, Department of Allergology, Clinical Immunology and Internal Diseases,

Collegium Medicum, Nicolaus Copernicus University, Bydgoszcz, Poland, phone: +48 501006104, e-mail: jendrek75@interia.pl

\begin{abstract}
Eosinophilic esophagitis (EoE) belongs to a broad group of allergic gastrointestinal diseases. More narrowly, it is classified as an eosinophilic gastrointestinal disease and is its most common representative. Until recently, this disease was extremely rare, but the last 3 decades have seen a rapid increase in its prevalence to such an extent that in some recent studies it has been named the most common esophageal disease after reflux disease. EoE is an interdisciplinary disorder on the borderline between gastrointestinal and allergologic diseases, requiring cooperation of these two branches of medicine in diagnostics and treatment. Despite the development of medical science it remains a poorly known disease, difficult to diagnose and even more difficult to treat. Recent years have seen the emergence of new studies and guidelines, both American and European, that have radically changed our approach to the diagnosis and treatment of EoE.
\end{abstract}

\section{Introduction}

Eosinophilic esophagitis (EoE) is a chronic immune-mediated esophageal disease characterized clinically by symptoms of esophageal dysfunction and histologically by eosinophilic infiltration of the esophageal wall. Other causes of esophageal eosinophilia have been excluded [1]. The prevalence of esophageal eosinophilia has risen rapidly within the last three decades to the point where it is now recognized as the second most common esophageal disorder after reflux disease. Between 1989 and 2009, its occurrence is estimated to have increased by more than 10 -fold, due to both heightened awareness among endoscopists and an actual growth in its incidence [2].

The etiology of EoE is not well understood. There is evidence to suggest its association with allergy as evidenced by:

- Coexistence of allergic conditions, such as asthma, allergic rhinitis, atopic dermatitis or food allergy in about $80 \%$;
- Peripheral eosinophilia in $\mathbf{5 0 - 7 0 \% ~ o f ~ c a s e s ; ~}$

- Elevated levels of total immunoglobulin E (IgE);

- Presence of specific IgE for inhalant allergens (in 90\% of adults) and food allergens (in $75 \%$ of children);

- Reduction or resolution of symptoms following elimination diet or steroid therapy [3].

The symptoms of EoE are not specific and may change with age. Feeding difficulties, food refusal as well as weight and growth retardation predominate in young children, whilst dysphagia and episodes of food trapping prevail in older children and adults [4].

In additional studies we find:

- Endoscopically: circular folds and rings of mucosa giving an image of esophageal trachealization, longitudinal furrows, papules, white plaques, obliteration of the vascular pattern, congestion and edema of the mucosa, and presence of esophageal strictures.

- Histologically: presence of eosinophils, widening of intercellular spaces, hypertrophy and elongation of papillae, and fibrosis of the basal layer of the mucosa. 
The diagnosis is based on histological examination. The criterion is the presence of more than 15 eosinophils per high power field (400x in an area of $\sim 0.3 \mathrm{~mm}^{2}$ or 60 eosinophils in an area of $\left.1 \mathrm{~mm}^{2}\right)$ [5].

\section{New diagnostic criteria}

The emergence of new US and European guidelines in recent years has dramatically changed the approach to the diagnosis and treatment of EoE $[5,6]$. The diagnosis of EoE currently consists of three steps:

- Step 1: EoE is suspected upon symptoms of esophageal dysfunction and endoscopic findings on the esophageal mucosa. The suspicion of EoE is strengthened by allergic coexistence.

- Step 2: Detection of at least 15 eosinophils/high-power field in esophageal biopsy specimens.

- Step 3: Differentiating EoE from other diseases that may cause esophageal eosinophilia (e.g. achalasia, Crohn's disease, connective tissue disease, drug hypersensitivity reaction) [7].

\section{Eosinophilic esophagitis - treatment}

Currently, 3 equivalent therapeutic options have been made available for the treatment of EoE since its inception: the use of proton pump inhibitors (PPIs), corticosteroids, and dietary treatment. Due to the lack of randomized trials comparing therapeutic strategies, the low cost of treatment, safety of therapy, convenience of use, and the good effect on reducing esophageal eosinophilia and endoscopic lesions, it is suggested that PPIs should be considered as first-line drugs, but treatment can also be started with steroid therapy or dietary treatment $[5,7]$.

\section{Proton pump inhibitors}

Until recently, PPIs only had an adjunctive role in the pharmacotherapy of EoE. In monotherapy, they were usually used in the early stages of the disease, as a test to differentiate EoE from gastroesophageal reflux disease (GERD) and from proton pump inhibitorresponsive esophageal eosinophilia (PPI-REE). Together with corticosteroids, they could be useful as adjunctive treatment in patients with coexisting EoE and GERD, whereas in patients with already diagnosed PPI-REE, they were the mainstay of treatment. However, recent studies have shown that PPI-REE and EoE are the same disease with different phenotypes. It is currently believed that acid reflux damages the epithelial barrier, allowing antigens to pass through it and initiate an allergic reaction resulting in eosinophilic infiltration. The main role of PPI treatment depends on their ability to regenerate the esophageal epithelial barrier and their recently demonstrated direct anti-inflammatory effect by reducing eotaxin 3 and Th2 mediators in the esophageal mucosa. Given these implications, in recent recommendations, experts have abandoned the use of PPI trial and PPP-REE diagnosis; however, they have recommended the use of PPI in patients with newly diagnosed EoE [7].

We treat EoE with omeprazole 20-40 mg twice a day or another PPI at an equivalent dose for 8 weeks, leading to clinical remission in $60 \%$ and histological remission in $50 \%$ of patients. Maintenance treatment for more than 1 year sustains remission in $73 \%$ of patients who initially responded to such treatment. When PPI therapy is discontinued, symptoms and/or eosinophilia usually return within 3-6 months. To date, the timing and doses of medication for maintenance therapy have not been clearly defined. The predominant approach is that the dose is gradually reduced to the lowest dose that keeps the disease in remission. Once reduced, it should be maintained for at least 1 year [5].

The effect of PPI therapy on the natural course of EoE remains unclear. As not all patients respond to PPI therapy, the search for a marker of response is ongoing. Patients with PPI-responsive EoE have been shown to have lower levels of Kir2.1 potassium channel gene expression (KCNJ2 gene), suggesting that some form of personalized therapy may be possible in the near future. Additionally, it has been demonstrated that patients with higher cytochrome CYP2C19 activity are at greater risk of losing EoE control despite continued PPI treatment [8].

\section{Glucocorticosteroids (GCSs)}

Both systemic and topical GCSs are used to treat EoE.

Systemic GCSs are effective histologically in $95 \%$ but clinically in $72 \%$. When they are discontinued, $45 \%$ relapse within 24 weeks. Long-term use is known to cause numerous adverse effects in $40 \%$ of patients, so they should be reserved for exceptional situations where rapid improvement is required or when local steroid therapy is ineffective.

Topical steroids are low in toxicity. They lead to histological improvement in $50-85 \%$ of cases and clinical improvement in $65 \%$. When they are discontinued, $45 \%$ of patients relapse after 24 weeks. However, what is important is that the side effects of esophageal candidiasis and asymptomatic adrenal suppression are clinically insignificant and rare (respectively in $15 \%$ and $10 \%$ of children on long-term treatment). The efficacy of topical GCSs in the treatment of EoE has been proven and they are currently being considered as first-line agents. GCSS inhibit IL-13-dependent pathways and genes, IL-13 being considered to be the key interleukin in patients with EoE, 
consequently leading to a reduction in esophageal eosinophilia and mast cell infiltration, a decrease in epithelial cell apoptosis, and a decrease in T-lymphocytes and pro-inflammatory cytokine levels. It is also known that topical GCSs can restore the epithelial barrier function and have a positive effect on tissue remodeling $[6,7]$.

Topical treatment includes formulations of inhaled corticosteroids (ICS), such as budesonide (2-4 mg/day in divided doses as a solution prepared from a suspension for nebulization $(0.5 \mathrm{mg} / \mathrm{ml})$ with the addition of $5 \mathrm{mg}$ of sucralose p.o.) or fluticasone (in children 88$440 \mu \mathrm{g}$ 2-4 times/day, in adults 440-880 $\mu \mathrm{g} 2$ times/ day), from a metered-dose inhaler without a spike in the form of applying the drug to the mouth while holding the breath and then swallowing it. Treatment should be long-lasting, even over 2 years [9].

It has been illustrated that the time of contact of a GCS with the esophageal mucosa is highly important in topical treatment. A longer contact time and higher esophageal deposition of GCS have been demonstrated for new "viscous" GCS formulations compared to oral inhalation. The shorter exposure time of inhaled steroids appears to be a major cause of EoE recurrence with long-term use [10]. In 2016, 2 new forms of budesonide were tested - an effervescent form of orally disintegrating tablets (BET) and a "sticky" budesonide suspension (BVS). The histological remission rates after 2 weeks were very high (94.7\%), but effervescent tablets were preferred by up to $80 \%$ of patients [11]. In the long term, 48-week treatment with $1 \mathrm{mg}$ of BET administered twice daily and $0.5 \mathrm{mg}$ of BET administered twice daily was effective in maintaining clinico-histological remission in $75.0 \%$ and $73.5 \%$, while side effects in the form of oral candidiasis were demonstrated in about $2-11 \%$ of subjects [12]. It has not been clearly defined how long and what dose of topical GCS should be used. It is known that discontinuation of steroid therapy in patients with controlled disease resulted in relapse in $81 \%$ of cases within an average of 22 weeks; therefore long-term treatment should be recommended. On the other hand, a 2-year follow-up of patients taking topical GSCs continuously showed maintenance of remission in up to $63 \%$ of cases. Additionally, a $50 \%$ reduction dose of fluticasone resulted in maintaining complete remission in $73 \%$ of cases. In view of the above-mentioned observations, it seems that treatment with these agents should be long-term (more than 2 years) and the prescribed dose ought to be the lowest effective dose so that the disease is maintained in remission $[9,11]$.

\section{Dietary treatment}

The type of diet in patients with EoE should be tailored to their individual needs. Dietary recommendations include consistency of meals, avoidance of food that is difficult to swallow, drinking more fluids during consumption, and increasing the chewing time. After dietary intervention and before any food introduction, clinical evaluation and gastroscopy with biopsy are necessary. In adults, additional pharmacotherapy is usually necessary [13].

Dietary treatment includes 3 options: empirical diet, targeted diet, and elemental diet.

\section{Empirical diet}

This is the most often used type of nutritional treatment. It involves exclusion of the 6 most common allergens: milk, eggs, fish/shellfish, nuts/peanuts, soy and wheat (SFED). This diet produces clinical and histologic improvement in $72 \%$ of cases. The dietary treatment is carried out for 8 weeks. After this period, remission usually occurs, which is confirmed by endoscopy with biopsy. Then, single allergens are repeated in the diet in order to select substances responsible for the symptoms, which is confirmed clinically and by endoscopic examination and biopsy evaluation [13].

The main problem with this diet is the high level of dietary restriction and the number of endoscopies. However, the study found that in the majority (65$85 \%$ ) of patients, the most frequent causative food was wheat, cow's milk, eggs and soy/legumes. Based on this, a diet excluding the 4 most common allergenic foods (FFED) was developed, leading to clinical and histopathological remission in $54 \%$ of adults and $71 \%$ of children. Additionally, it was reported that in as many as $50 \%$ of adults and $74 \%$ of children, the trigger was either cow's milk or wheat. Therefore, a diet excluding the 2 most commonly allergenic foods (TFED) was developed - this approach led to remission in up to $40 \%$ of patients. Compared with starting the dietary treatment with SFED, this step-up strategy reduced the number of endoscopic procedures and the time of the diagnostic process by as much as 35\% [14].

\section{Targeted diet}

This diet excludes allergens indicated by a positive prick or patch skin tests or elevated slgE. The efficacy of such management in adults is about $32 \%$, and in children $45 \%$ [15].

Due to the fact that allergy testing does not predict response to dietary treatment well, new strategies are under development. Two of them show promising results:

- measurement of food-specific IgG4 in esophageal biopsy as an indicator of response efficacy following elimination diets. High specificity of this procedure has been demonstrated, but its sensitivity was insufficient with respect to peanut and soy; 
- lymphocyte proliferation test on patient serum in addition to the previous test. A positive result in both of these tests resulted in concordance between the tests and results of the elimination diet ranging from $53 \%$ to $75 \%$ [16].

An even more invasive approach was reported by the Amsterdam group. This group study involved puncturing the esophageal mucosa during endoscopy and administering an extract of the allergen directly into the esophageal mucosa. An immediate type reaction was observed in 5 of 8 and a delayed type reaction in 2 out of 8 patients. It has not yet been determined whether these reactions are correlated with the results of elimination diets [16].

\section{Elemental diet}

In the elemental diet, all food allergens are eliminated by giving the allergic person industrially obtained amino acid mixtures. It is highly effective (histopathological remission in up to $90 \%$ of cases), but has numerous disadvantages. Therefore, it should be used only in exceptional situations, such as failure of empirical elimination diet, young children, and when inflammation persists, especially if rapid clinical improvement is required [13].

As there are no controlled studies/research, the choice of treatment is individualized and based on patient preference. The dietary attitude requires strong motivation both of a patient and physician, as well as the assistance of a dietitian. If food trigger is identified, it is fully avoided. The long-term efficacy of this approach has been demonstrated in a 4-year clinical follow-up $[6,7,13]$.

\section{Esophageal dilatation}

It is primarily recommended for patients with dysphagia accompanied by esophageal stenosis less than $13 \mathrm{~mm}$. It is an effective and safe procedure. The efficacy in adults is approximately $75 \%$, and the risk of esophageal perforation is low at approximately $1 \%$ [17].

\section{Azathioprine and 6-mercaptopurine, montelukast, cromones}

Only one article evaluating the efficacy of azathioprine or 6-mercaptopurine (6-MP) in EoE has been published. These drugs had a positive steroid-sparing effect and on induction and maintenance of long-term remission without GCS in 3 cases of steroid-dependence in adults. In contrast, there is insufficient evidence to state that montelukast and cromones have no effect on either symptoms or eosinophilic infiltration in patients with EoE [18].

\section{Biological treatment}

Potential future role include treatment of steroid-resistant patients, maintenance of steroid-induced remission, and treatment of EoE patients with coexisting atopic diseases.

Treatment with anti-IL-5, anti-IgE, anti-TNF- $\alpha$

Mepolizumab and reslizumab (anti-IL-5 antibodies) have no effect on symptoms and slightly reduce esophageal eosinophilia. Omalizumab (anti-lgE antibody) and infliximab (anti-TNF- $\alpha$ antibody) have no effect on either symptoms or esophageal eosinophilia. In studies, benralizumab, an anti-IL-5 $\alpha$-chain antibody, has normalized gastrointestinal eosinophilia in hypereosinophilic syndrome, but has not yet been analyzed in terms of EoE $[19,20]$.

\section{Anti-IL-13 treatment}

IL-13 is a key mediator in the pathogenesis of EoE. To date, two monoclonal antibodies against IL-13 have been tested, QAX576 and RPC4046. QAX576 showed a statistically significant decrease in esophageal eosinophilia, but had no effect on clinical symptoms. Examination of RPC4046 showed a significant reduction in esophageal eosinophilia and a strong (but non-significant) tendency to reduce dysphagia symptoms. What is crucial in this study is the fact that half of the included patients were previously refractory to topical steroids [21].

\section{Anti-IL-13 and IL-4 treatment}

The greatest hope for successful treatment of EoE is associated with dupilumab. Similarly to IL-13, IL-4 has also shown to be a key IL in patients with Th2-dependent disease. Dupilumab antagonizes IL-4 and IL-13 by the $\alpha$ subunit, which is common for both IL-13 and IL-4 receptors. 12-week treatment with dupilumab showed a considerable clinical, endoscopic, and histologic improvement [22].

\section{Biological treatments - the future}

Currently, there are some studies for new treatments based on other immunocompetent molecules. Some hope lies within the following molecules:

- OC000459 (CRTH2 - a homolog of the Th2 cell receptor antagonist) - administered at a dose of $100 \mathrm{mg}$ over 8 weeks significantly reduced eosinophilic infiltration. However, it had no effect on clinical symptoms or endoscopic changes. Because its efficacy is lower than that of steroids, it was excluded from further studies [23].

- Integrin $\alpha 4 / \beta 7$ is involved in the passage of lymphocytes. Vedolizumab is an antibody of $\alpha 4 / \beta 7$ integrin. 
Its potential role in the treatment of EoE was suggested by 2 case studies of patients with coexisting inflammatory bowel disease in which treatment resulted in clinical and histological responses on the esophageal side. In addition, its antieosinophilic activity was demonstrated in 2 case series involving eosinophilic gastroenteritis [24].

- AK002 (anti Siglec-8 antibody) in a mouse model is responsible for reduction in IL-5-induced eosinophilia. A phase II study of AK002 in patients with eosinophilic gastroenteritis demonstrated reduction of symptoms and gastrointestinal tissue eosinophils. Data on the efficacy of AKO02 in patients with EoE are not currently available [25].

- Tezepelumab (anti-TSLP antibody) reduces esophageal eosinophilia and food response inflammation in a mouse model of EoE. Tezepelumab has proved to be effective in patients with severe asthma, but has not yet been studied in patients with EoE [26].

Other drugs and immunocompetent molecules:

There is also a theoretical basis for the use of drugs from other groups, such as:

- Losartan: blocks transforming growth factor- $\beta$ (TGF- $\beta$ )-dependent signaling, which is crucial for eosinophil recruitment and plays an important role in tissue remodeling in patients with EoE. Therefore, losartan is currently under investigation for the treatment of EoE and eosinophilic gastroenteritis [27].

- Verapamil: has recently been shown to attenuate IL4-induced eotaxin expression in esophageal epithelial cells. Therefore calcium channels are a potential target for reducing the recruitment of esophageal eosinophilia. However, there are no clinical data on such treatment [28].

- AXP1275, a CCR-3 antibody against eotaxin, which is a key factor in the recruitment of eosinophils to the esophageal mucosa. It has been illustrated in a mouse model that this antibody inhibits eotaxin-dependent eosinophil influx and mucosal damage in patients with eosinophilic gastroenteritis. However, studies in patients with asthma have not demonstrated the efficacy of AXP1275 and studies in patients with EoE have not yet been performed [29].

In addition, it is believed that a future point of treatment may be antibodies against the following interleukins:

- IL-9: seems to be a major factor which is responsible for mast cell recruitment and damage of the epithelial barrier in patients with EoE. Until now antibody against IL-9 has been tested in patients with asthma, but has not yet been studied in patients with EoE.

- IL-15: has been shown to mediate EoE pathogenesis in in vivo models. An antibody against IL-15 (CALY-002) is currently under investigation. Clinical data regarding its efficacy in the treatment of EoE are not yet available.

- IL-33: increased expression of endogenous IL-33 has been shown to be associated with EoE development in children, and exogenous IL-33 promotes EoE development in mice. However, to date, there are no clinical data on the use of anti-IL-33 antibodies in patients with EoE [30].

\section{Summary}

To effectively diagnose, treat, and prevent complications of EoE, we must have proven procedures and effective medications in place. To achieve this, there is a need to fill the gaps in our knowledge of this disease. These gaps and deficiencies include aspects, such as the use of uniform endpoints in clinical trials, direct studies comparing single therapies and combinations of therapies, accurate data on the natural history of EoE, and mechanisms and management of persistent symptoms despite histological remission. It is also important to conduct long-term studies to evaluate the effectiveness of maintenance treatment, learn about new biomarkers in the diagnosis and monitoring of EoE, and validate non-endoscopic methods for monitoring EoE activity. We should also understand the impact of the history of food exposure and avoidance of environmental allergens on the development and natural history of EoE, and assess the interactions between oral immunotherapy and the development of EoE, as well as the impact of atopic diseases on the development and progress of EoE.

Thus, in view of the above, it seems that the road to the full knowledge of this disease may still be long [7].

\section{Conflict of interest}

The authors declare no conflict of interest.

\section{References}

1. Park H. An overview of eosinophilic esophagitis. Gut Liver 2014; 8: 590-7.

2. Hruz P. Epidemiology of eosinophilic esophagitis. Dig Dis 2014; 32: 40-7.

3. Roy-Ghanta S, Larosa D, Katzka D. Atopic characteristics of adult patients with eosinophilic esophagitis. Clin Gastroenterol Hepatol 2008; 6: 531-5.

4. Straumann A, Aceves S, Blanchard C, et al. Pediatric and adult eosinophilic esophagitis: similarities and differences. Allergy 2012; 67: 477-90.

5. Lucendo A, Molina-Infante J, Arias A, et al. Guidelines on eosinophilic esophagitis: evidence-based statements and recommendations for diagnosis and management in children and adults. United European Gastroenterol J 2017; 5: 335-58.

6. Hirano I, Chan E, Rank M, et al. AGA institute and the joint task force on allergy-immunology practice parameters clinical 
guidelines for the management of eosinophilic esophagitis. Ann Allergy Asthma Immunol 2020; 124: 416-23.

7. Dellon E, Liacouras C, Molina-Infante J, et al. Updated International Consensus Diagnostic Criteria for Eosinophilic Esophagitis: Proceedings of the AGREE Conference. Gastroenterology 2018; 155: 1022-33.

8. Gonsalves N, Aceves S. Diagnosis and treatment of eosinophilic esophagitis. J Allergy Clin Immunol 2020; 145: 1-7.

9. Dohil R, Newbury R, Fox L, et al. Oral viscous budesonide is effective in children with eosinophilic esophagitis in a randomized placebo-controlled trial. Gastroenterology 2010; 139: 418-29.

10. Greuter T, Safroneeva E, Bussmann C, et al. Maintenance treatment of eosinophilic esophagitis with swallowed topical steroids alters disease course over a 5-years follow-up period in adult patients. Clin Gastroenterol Hepatol 2019; 17: 419-29.

11. Miehlke S, Hruz P, Vieth $M$, et al. A randomised, double-blind trial comparing budesonide formulations and dosages forshort-term treatment of eosinophilic oesophagitis. Gut 2016 65: 390-9.

12. Lucendo A, Miehlke S, Schlag C, et al. Efficacy of budesonide orodispersible tablets as induction therapy for eosinophilic esophagitis in a randomized placebo-controlled trial. Gastroenterology 2019; 157: 74-86e15.

13. Arias A, Gonzalez-Cervera J, Tenias J, et al. Efficacy of dietary interventions for inducing histologic remission in patients with eosinophilic esophagitis: a systematic review and meta-analysis. Gastroenterology 2014; 146: 1639-48.

14. Molina-Infante J, Modolell I, Alcedo J, et al. Step-up empiric elimination diet for pediatric and adult eosinophilic esophagitis: the 2-4-6 study. United Eur Gastroenterol J 2016; (Suppl 5): A126.

15. Dellon S, Guo R, McGee S, et al. An allergen-specific immune signature identifies food triggers in eosinophilic esophagitis with high accuracy. Gastroenterology 2018; 154: S260.

16. Warners M, Terreehorst I, van den Wijngaard R, et al. Abnormal responses to local esophageal food allergen injections in adult patients with eosinophilic esophagitis. Gastroenterology 2018; 154: 57-60e2.

17. Moawad F, Cheatham J, DeZee K. Meta-analysis: the safety and efficacy of dilation in eosinophilic oesophagitis. Aliment Pharmacol Ther 2013; 38: 713-20.

18. Netzer P, Gschossmann JM, Straumann A, et al. Corticosteroid-dependent eosinophilic oesophagitis: azathioprine and 6-mercaptopurine can induce and maintain long-term remission. Eur J Gastroenterol Hepatol 2007; 19: 865-9.

19. Markowitz J, Jobe L, Miller M, et al. Safety and efficacy of reslizumab for children and adolescents with eosinophilic esophagitis treated for 9 years. J Pediatr Gastroenterol Nutr 2018 66: 893-7.

20. Kuang F, Legrand F, Makiya M, et al. Benralizumab for PDGFRA-negative hypereosinophilic syndrome. N Engl J Med 2019; 380: 1336-46.

21. Hirano I, Collins M, Assouline-Dayan Y, et al. RPC4046, a monoclonal antibody against IL13, reduces histologic and endoscopic activity in patients with eosinophilic esophagitis. Gastroenterology 2019; 156: 592-603e10.
22. Hirano I, Dellon E, Hamilton J, et al. Efficacy of dupilumab in a phase 2 randomized trial of adults with active eosinophilic esophagitis. Gastroenterology 2020; 158: 111-22.

23. Straumann A, Hoesli S, Bussmann C, et al. Antieosinophil activity and clinical efficacy of the CRTH2 antagonist OC000459 in eosinophilic esophagitis. Allergy 2013; 68: 375-85.

24. Nhu Q, Chiao H, Moawad F, et al. The Anti-a4b7 integrin therapeutic antibody for inflammatory bowel disease, vedolizumab, ameliorates eosinophilic esophagitis: a novel clinical observation. Am J Gastroenterol 2018; 113: 1261-3.

25. Youngblood B, Brock E, Leung J, et al. Siglec-8 antibody reduces eosinophil and mast cell infiltration in a transgenic mouse model of eosinophilic gastroenteritis. JCI Insight 2019; 4: e126219.

26. Noti M, Wojno E, Kim B, et al. Thymic tromal lymphopoietin-elicited basophil responses promote eosinophilic esophagitis. Nat Med 2013; 19: 1005-13.

27. Cheng K, Gupta S, Kantor S, et al. Creating a multi-center rare disease consortium - the Consortium of Eosinophilic Gastrointestinal Disease Researchers (CEGIR). Transl Sci Rare Dis 2017; 2: 141-55.

28. Odiase E, Zhang X, Chang Y, et al. L-type calcium channel inhibitors (verapamil and diltiazem) block Th2-cytokine-stimulated eotaxin-3 secretion in esophageal squamous cells from patients with eosinophilic esophagitis. Gastroenterology 2019; 156 (Suppl 1): S39.

29. Song D, Shim M, Lee N, et al. CCR3 monoclonal antibody inhibits eosinophilic inflammation and mucosal injury in a mouse model of eosinophilic gastroenteritis. Allergy Asthma Immunol Res 2017; 9: 360-7.

30. Greuter T, Hirano I, Dellon E. Emerging therapies for eosinophilic esophagitis. J Allergy Clin Immunol 2020; 145: 38-45.

Received: 5.02.2021

Accepted: 21.02 .2021 